\title{
Toroidal Carbon Nanotubes with Encapsulated Atomic Metal Loops
}

\author{
Mark T. Lusk and Nathaniel Hamm* \\ Department of Physics, Colorado School of Mines
}

(Dated: July 31, 2007)

\begin{abstract}
Toroidal carbon nanotubes can serve as hosts for encapsulated loops of atomic metal wires. Such composite structures have been analyzed using density functional theory for a semiconducting $\mathrm{C}_{120}$ torus encapsulating chains of $\mathrm{Fe}, \mathrm{Au}$ and $\mathrm{Cu}$ atoms. The sheathed metal necklaces form a zigzag structure and drops the HOMO/LUMO bandgap to less than $0.1 \mathrm{eV}$. The iron composite is ferromagnetic with a magnetic moment essentially the same as that of bcc iron. The azimuthal symmetry of these toroidal composites suggests that they may offer novel elecromagnetic properties not associated with straight, metal-encapsulated carbon nanotubes.
\end{abstract}

\section{INTRODUCTION}

Fullerene-based toroids combine the novel characteristics of carbon nanotubes with electromagnetic and mechanical properties unique to closed-ring geometries. An intriguing possibility, examined in this paper and shown in Figure 1, is that atomic chains of metal can be encapsulated within such toroidal carbon nanotubes (TCNs). The carbon torus would serve as a stabilizing sheath for the atomic metal necklaces resulting in rigid, stable structures at finite temperatures with novel paramagnetic or ferromagnetic properties properties. Beyond applications that exploit the properties of independent rings, assemblies of such ferromagnetic tori might be expected to selfassemble into columnar structures with unusual macroscopic behavior. The electronic structure and mechanical stability of these robust atomic necklaces are investigated using density functional theory (DFT) with the objective of establishing ground state geometries, binding energies and magnetic moments.

At least four types of single-wall fullerene toroids have been considered since the idea was first proposed fifteen years ago[1. Two variations of Type $1 \mathrm{TCNs}$ exploit the curvature induced by pentagonal and septagonal defects to generate polygonal graphitic tori [1, 2 2 and rings as small as $C_{120}$ have been considered [3]. The symmetric Type 2 TCNs are toroidal polyhexes 4 created by joining the ends of single-wall carbon nanotubes [5]. Perhaps the most technologically promising category of toroid is derived from $C_{60}$ molecules that are joined with pentagonal and septagonal rings and looped into undulated rings [6], [7. These are referred to here as Type 3 toroids. Finally, Haeckelite tubules 8 , based on a two-dimensional bravais lattice with 5, 6 and 7-member rings, can also be joined at the ends to make the Type 4 TCNs 6, [7. The first two types of TCNs are theoretical constructs that are thought to be mechanically stable. It has been estimated that TCNs of Type 1 are in fact more stable than $\mathrm{C}_{60}$ molecules[3], while Type 2 TCNs are expected to be stable provided they have a sufficiently large toroidal diameter [5], [9]. Carbon toroids of Types 3 and 4 , on the other hand, have been realized experimentally and are demonstrably stable $[6,[7]$.
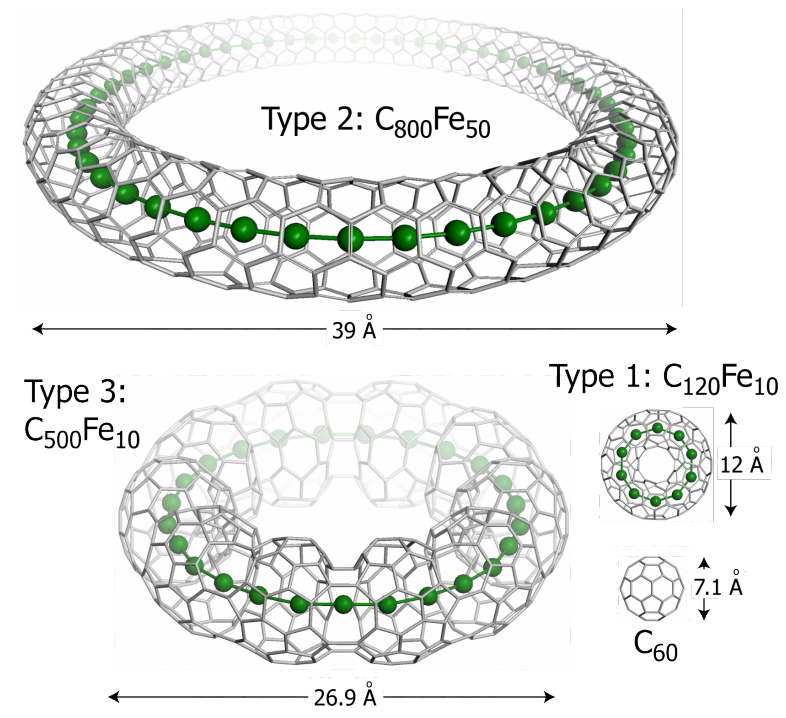

FIG. 1: Atomic chains of Fe atoms encapsulated within three types of toroidal carbon nanotubes. A bucky ball is shown at lower right for size comparison. All structures are drawn to scale.

For all toroids, delocalized $\pi$ electrons can be induced into ballistic, persistent motion by the application of a magnetic field perpendicular to the plane of the torus. The resulting orbital magnetic response of tori can be substantial and has been investigated using tight binding theory with the magnetic vector potential accounted for using London theory 10, , 11]. Type 1 toroids are predicted to exhibit a large diamagnetic response [12, [13, [5, while a class of conducting Type 2 TCNs are expected to be able to generate colossal paramagnetic moments [14, [5, 15]. More recently, carbon toroids of Types 3 and 4 TCNs have been shown experimentally to have paramagnetic behavior consistent with tight binding model predictions [6]. The septagonal and pentagonal rings in these structures are predicted to result in ferromagnetic behavior at sufficiently low temperatures in the absence of any flaws. All types of TCNs are expected to exhibit Aharanov-Bohm effects[13].

Paralleling activity associated with toroidal fullerene 
structures have been advances in the fabrication and analysis of isolated atomic chains of metal atoms. Metal contacts created with a scanning tunneling microscope can be slowly pulled apart to extrude an atomic chain of $\mathrm{Au}$ atoms 16, 17. Stable monatomic chains of $\mathrm{Au}$ atoms can support currents of $80 \mu A$-clear evidence of ballistic transport in this alternate manifestation of one-dimensional conductors [16-and the quantum conductance in these metallic atomic chains has also been measured[17]. As subsequent DFT analysis of atomic chains of Au atoms, intended to address observed anomalies, indicated that zigzag structures are more stable than a linear chains as a result of reduced transverse kinetic energy of electrons 18. Such configurations are most likely metastable since previous investigations have identified polyhedral ground state structures for small clusters of $\mathrm{Fe}$ and $\mathrm{Au}$ atoms 19, 20.

Iron structures sheathed within straight single wall carbon nanotubes have been studied both computationally and experimentally 21, 22]. A DFT analysis of atomic chains of $\mathrm{Fe}$ within single-wall carbon nanotubes concluded that the spacing between Fe atoms and their nearest $\mathrm{C}$ neighbors determined the character of the $\mathrm{Fe}$ wire 21]. The Fe-C bond length of $1.9 \AA$ associated with $(3,3)$ tubes resulted in a composite with semi-conductor character and no magnetic moment. On the other hand, an Fe-C bond length of $2.1 \AA$ associated with $(4,4)$ tubes resulted in a composite with the metallic character and magnetic moment of $2.6 \mu_{B}$ per atom 21. The geometry of such tubes precludes the generation of axial persistent currents and a colossal paramagnetic response, but they might be used to help predict the conduction and ferromagnetic nature wires encapsulated within TCNs.

The present work brings together the unusual behavior of toroidal carbon nanotubes and atomic metallic chains within a simple setting: a Type $1 \mathrm{C}_{120}$ torus with $\mathrm{D}_{5 d}$ symmetry 2] encapsulating closed, monatomic chains of $\mathrm{Au}, \mathrm{Fe}$, and $\mathrm{Cu}$ atoms. The iron necklace is of particular importance because of the possibility that it has a ferromagnetic nature at finite temperatures. The gold necklace was also considered, though, because of the focus that $\mathrm{Au}$ has received in atomic metal wires [16, [17]. Analysis was $\mathrm{Cu}$ atoms was thought to offer an intermediate composite since it is a noble metal, like $\mathrm{Au}$, and a Period 4 metal like Fe. The Type 1 TCN chosen for analysis is a polygonal $\mathrm{C}_{120}$ torus with $D_{5 d}$ symmetry-chosen for its predicted stability and minimal size 2]. The $\mathrm{C}_{120}$ torus is composed of an inner surface of ten septagons and an outer surface of ten pentagons patched with twenty hexagons as shown in Figure 2. Encapsulated atomic loops composed of ten metal atoms were considered in order to exploit maintain the computational expedient of $\mathrm{D}_{5 d}$ symmetry while creating $\mathrm{M}-\mathrm{M}$ bond lengths on the order those calculated for isolated metal chains. Density functional theory (DFT) was used to determine the ground state configurations and to quantify M-M bond
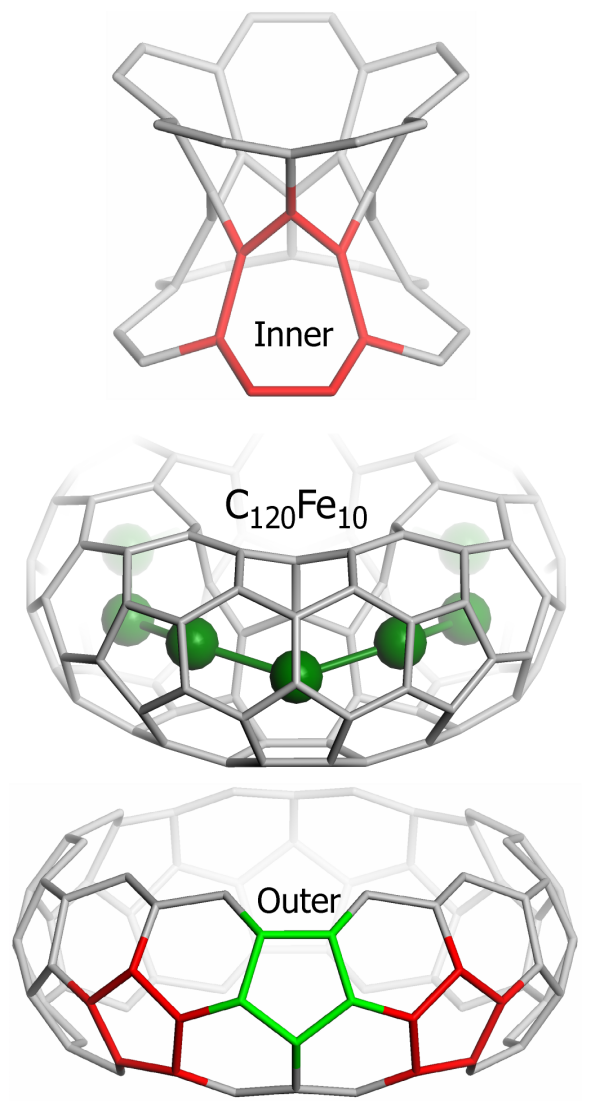

FIG. 2: Break-out view of the inner and outer structures of the $\mathrm{C}_{120} \mathrm{Fe}_{10}$ molecule with shading used to highlight polygonal defect types.

length, minimum $\mathrm{C}-\mathrm{M}$ bond length, magnetic moment, density of states, conduction character, binding energy of the encapsulated metal atoms.

\section{METHOD}

All calculations were performed with the real-space, numerical atomic orbital, DFT code, $\mathrm{DMOL}^{3}[23$. A norm conserving, spin unrestricted, semi-core pseudopotential approach was employed with electron correlation accounted for using the Perdew-Wang generalized gradient approximation (GGA) 24]. Double numerical basis sets were used with $4 p$ polarization functions for Fe atoms and $3 d$ polarization functions for $\mathrm{C}$ atoms. A smearing value of $0.272 \mathrm{eV}$ was used for all structures.

As a check on the method, an Fe dimer was determined to have a $\left({ }^{7} \Delta_{u}\right)$ collinear ferromagnetic ground state with a bond length of $2.00 \AA$ and magnetic moment of $7.0 \mu_{B}$ by Hirshfeld analysis-consistent with both experimental and other DFT results 25. As a further check, the atomic spacing, binding energy and magnetic moment of bcc Fe was evaluated to be $2.48 \AA,-4.89 \mathrm{eV}$ and and $2.22 \mu_{B^{-}}$ 


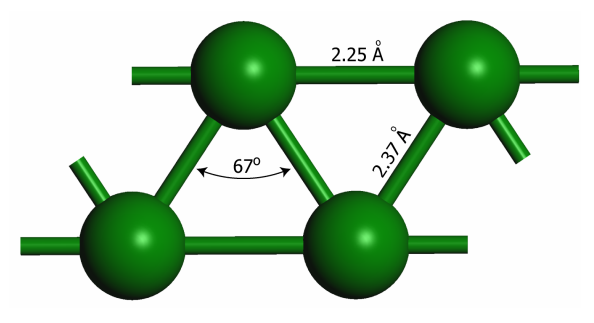

FIG. 3: Ground state structure for a periodic chain of $\mathrm{Fe}$ atoms.

reasonable matches to the experimental values of 2.47 $\AA,-4.32 \mathrm{eV}$ and $2.22 \mu_{B}$ per atom 26, 27, 28. DFT values of $2.76 \mathrm{eV}$ and $2.08 \mu_{B}$ per atom were obtained elsewhere 29].

\section{RESULTS}

\section{Free Standing Metal Chains}

Periodic structures of pairs of free standing $\mathrm{Fe}, \mathrm{Au}$ and $\mathrm{Cu}$ atoms were analyzed in order to understand the character of metallic chains prior to encapsulating them in carbon toroids. The ground state configuration for the Au chain was found to be a zigzag structure with a bond length of $2.83 \AA$ and an angle between adjacent atoms of $57^{\circ}$. There is also a local equilibrium of higher energy at $134^{\circ}$, and this is close to that obtained by Sánchez-Portal et al. who identified an equilibrium angle of $131^{\circ}[18$. Analogous results, summarized in Tables 2 and 3, were obtained for $\mathrm{Fe}$ and $\mathrm{Cu}$ chains, and all three chains exhibit ground state bending angles of approximately $60^{\circ}$; an affinity for symmetric, three-atom bonding is optimum within the geometric constraints imposed. As shown in Figure 3, such structures amount to two-dimensional, triangular lattices structures rather than linear chains. Key property data is summarized in the tables. All positive eigenvalues of the Hessian matrices associated with periodic cells of four atoms indicate at least linearized stability at $0 K$ for all three chains. A Jahn-Teller distortion, manifested in both bond angle and length, was noted for metallic strands of eight atoms or more, and this is consistent with previous ab initio investigations [30].

\section{Atomic Metal Wires within SWCNT}

Single-strand atomic wires of metal atoms encapsulated within single-wall carbon nanotubes (SWCNT) are of interest on their own and can be used to anticipate the electronic structure and stability of wires encapsulated within carbon tori. The tube diameter of the $\mathrm{C}_{120}$ torus is between that of $(3,3)$ and $(4,4)$ SWCNT, and
(3,3)-Fe SWCNT

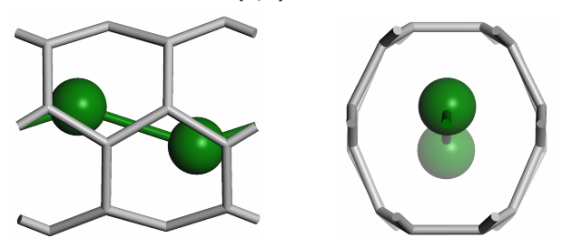

$(4,4)$-Fe SWCNT

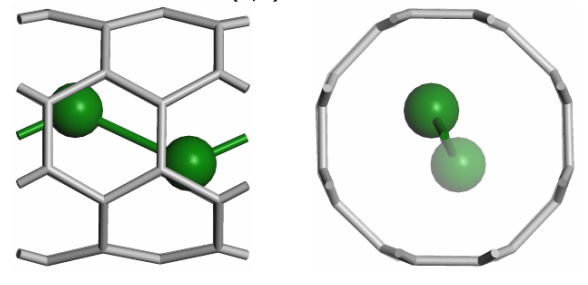

FIG. 4: Ground state structures of $(3,3)$ and $(4,4)$ SWCNT embedded with atomic Fe wire.

so these fullerenes were chosen for more detailed analysis with metal wires. Previous DFT studies considered atomic wires of Fe within SWCNT[21, 31]. The analysis for $(n, n)$ tubes considered only one metal atom per unit cell, though, and therefore did not allow for the possibility of the metal chain forming a zigzag configuration. Since the toroidal structure should allow for such corrugation, the SWCNT analysis was performed for doublecell, periodic structures with both $\mathrm{Fe}, \mathrm{Au}$ and $\mathrm{Cu}$ wires. The resulting equilibrium configurations for are shown in Figures 4,5 and 5 with property data summarized in Tables 1 through 3. As expected, the encapsulated wires do exhibit a zigzag structure although the carbon sheath flattens the wires relative to their free standing configuration. For the $(4,4)$ tube, the Fe wire causes a diametral tube strain of $0.94 \%$ while the Au wire causes a larger diametral expansion with $2.44 \%$. Reassuringly, the $\mathrm{Cu}$ wire causes a dilation between these two-2.1\%. In the case of the smaller $(3,3)$ tube, the diametral expansion of the $\mathrm{Au}$ tube is $8.7 \%$, while the Fe tube shows a non-uniform distortion with bi-lateral strains of $8.2 \%$ and $-5.2 \%$. The $\mathrm{Cu}$ tube is uniformly dilated, like Au case, with a strain of $3.4 \%$. The allowance for zigzagging within the tubes significantly impacts the electronic structure of the composite. If a periodic cell half as long is considered, so that there is only one Fe atom per period, the bandgap is $0.87 \mathrm{eV}$ instead of $0.48 \mathrm{eV}$. The first number is consistent with the $0.88 \mathrm{eV}$ obtained previously [31]. The magnetic moment of both straight and zigzagging chains of Fe atoms were extinguished within $(4,4)$ CNTs. An earlier analysis on straight, sheathed chains reported a magnetic moment of $2.6 \mu_{B}$ per Fe atom 31 . 


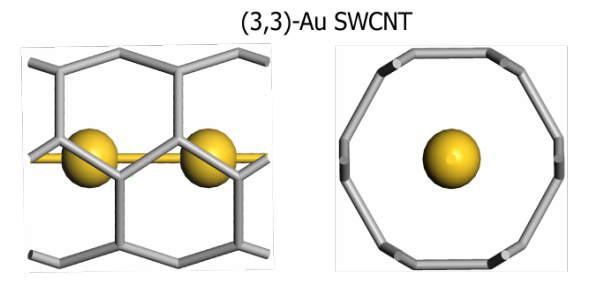

(4,4)-Au SWCNT

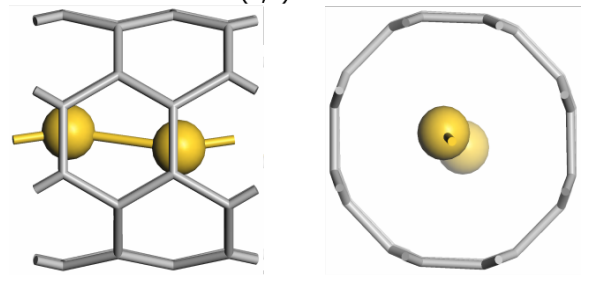

FIG. 5: Ground state structures of $(3,3)$ and $(4,4)$ SWCNT embedded with atomic $\mathrm{Au}$ wire.

(3,3)-Cu SWCNT

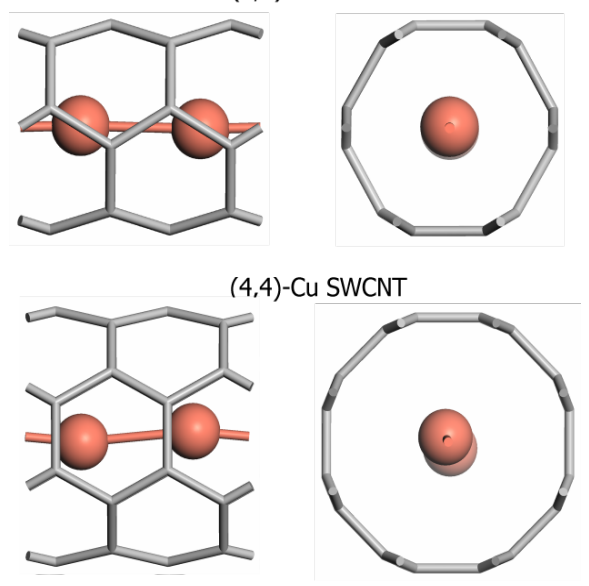

FIG. 6: Ground state structures of $(3,3)$ and $(4,4)$ SWCNT embedded with atomic $\mathrm{Cu}$ wire.

\section{$\mathbf{C}_{120}$ Torus}

The $\mathrm{C}_{120}$ torus was first analyzed in the absence of atomic wire, and the energy per $\mathrm{C}$ atom was found to be less than that of $\mathrm{C}_{60}$ by $0.09 \mathrm{eV}$ (smearing) and $0.16 \mathrm{eV}$ (no smearing). This is in contrast to an earlier tight binding calculation which predicted that the toroidal state would be of lower energy 2]. The difference is small, though, and the $\mathrm{C}_{120}$ torus should be very stable. A reactive molecular dynamics code 9] was used to estimate that the structure is stable to at least $2000 \mathrm{~K}$. The torus has no intrinsic magnetic moment and exhibits a bandgap of $0.91 \mathrm{eV}$ between its highest occupied and lowest unoccupied molecular orbitals (HOMO/LUMO). For comparison, the HOMO/LUMO bandgap of $\mathrm{C}_{60}$ was found to be $1.5 \mathrm{eV}$-close to the experimental measurement of $1.6 \mathrm{eV} 32$.

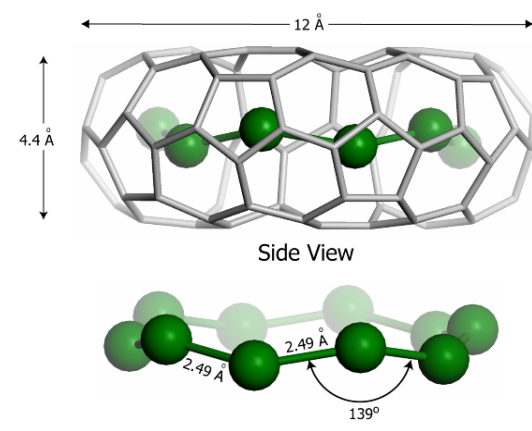

Perspective View of Fe Chain

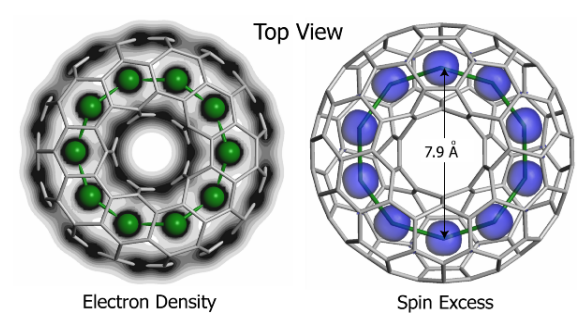

FIG. 7: Ground state structure of $\mathrm{C}_{120} \mathrm{Fe}_{10}$. The axial positions of the $\mathrm{Fe}$ atoms alternate between $\pm 0.21 \AA$. Also shown are the electron density cross-section (center) and spin excess isosurface (far right).

\section{Atomic Metal Necklaces within a $\mathbf{C}_{120}$ Torus}

The ground state was obtained for $\mathrm{M}_{10}$ loops encapsulated within the $\mathrm{C}_{120}$ torus with the resulting structures shown in Figures 7, 8 and 9. The associated properties are summarized in Tables 1-3. Consistent with the ground state morphology of free standing metallic chains, the encapsulated necklace of Fe atoms forms a zigzag structure which is highlighted in the bottom of the figure. The minimum Fe-C bond length was found to be between that of the $(3,3)$ and $(4,4)$ SWNTs. The toroidal composite has a HOMO/LUMO bandgap of less than $0.1 \mathrm{eV}$ and has a magnetic moment of $2.28 \mu_{B}$ per atom-approximately the same as that associated with bcc Fe. The spatial distribution of this magnetic moment is shown in Figure 7 through an isosurface plot of the spin excess. At zero temperature, the Fe atoms have aligned spin axes and so the composite is ferromagnetic. In contrast, neither of the straight nanotubes with $\mathrm{Fe}$ showed any intrinsic moment because of stronger Fe-C bonding for the tubes.

Encapsulation of an $\mathrm{Au}_{10}$ necklace was found to be energetically unfavorable, while the $\mathrm{Cu}_{10}$ encapsulation is an energetically neutral process. As indicated in Tables 2 and 3, these results are consistent with those associated with CNT encapsulations.

The DFT investigation indicates that the $\mathrm{C}_{120}$ torus with all three types of metal necklaces is structurally stable at zero temperature. The TCN has a strong affin- 


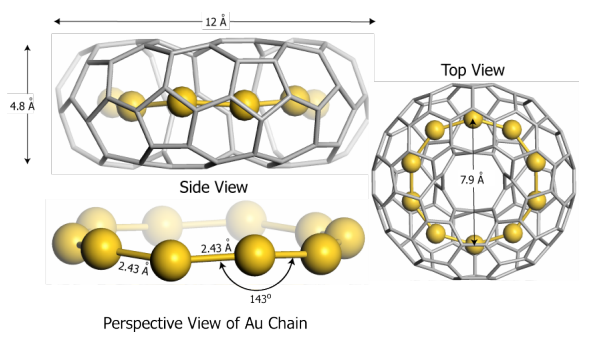

FIG. 8: Ground state structure of $\mathrm{C}_{120} \mathrm{Au}_{10}$. The axial positions of the $\mathrm{Au}$ atoms alternate between $\pm 0.076 \AA$. Also shown are the electron density cross-section (center) and spin excess isosurface (far right).

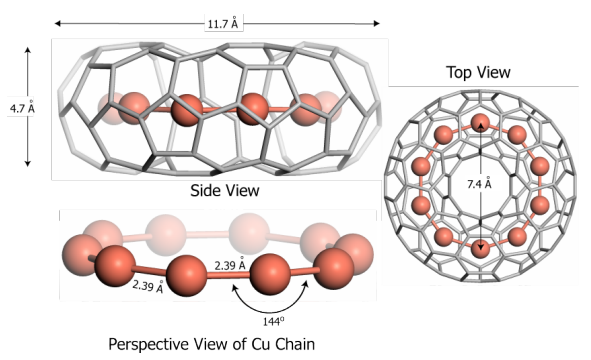

FIG. 9: Ground state structure of $\mathrm{C}_{120} \mathrm{Cu}_{10}$. The axial positions of the $\mathrm{Cu}$ atoms alternate between $\pm 0.025 \AA$. Also shown are the electron density cross-section (center) and spin excess isosurface (far right).

ity for $\mathrm{Fe}$ atoms $(1.82 \mathrm{eV} / \mathrm{Fe})$ and a neutral affinity for $\mathrm{Cu}$ atoms, but the placement of a $\mathrm{Au}$ necklace within the torus would require an energy input of $5.54 \mathrm{eV}$ per $\mathrm{Au}$ atom. This precludes the study of $\mathrm{Au}$ wires within this setting. The $(3,3)$ and $(4,4)$ CNTs can be used to estimate the affinity of $\mathrm{C}_{120}$ toroids for metal atoms; the binding energy was found to be midway between the CNT values for each of the three metal chains. This allowed the prediction, in advance, that $\mathrm{C}_{120}$ would exhibit a neutral affinity for the $\mathrm{Cu}$ necklace. The presence of the metal atoms causes the TCN composite to have a HOMO/LUMO bandgap of less than $0.1 \mathrm{eV}$ in all three cases. When encapsulated with an Fe necklace, the torus exhibits ferromagnetic properties close to that of

\begin{tabular}{||l||c|c|c|c|c|c||}
\hline \hline Structure & $\begin{array}{c}\mathrm{M}-\mathrm{M} \\
\AA\end{array}$ & $\begin{array}{c}\mathrm{M}-\mathrm{C} \\
\AA_{\text {min }}\end{array}$ & $\begin{array}{c}\mathrm{M}-\mathrm{M} \\
\text { Angle }\end{array}$ & $\begin{array}{c}\text { Gap } \\
\mathrm{eV}\end{array}$ & $\begin{array}{c}\text { Mom. } \\
\mu_{B} / \mathrm{M}\end{array}$ & $\begin{array}{c}\mathrm{E}_{\mathrm{B}} \\
\mathrm{eV} / \mathrm{M}\end{array}$ \\
\hline Fe bcc & 2.49 & - & - & 0.00 & 2.22 & 4.89 \\
\hline Fe chain & 2.35 & - & $59^{\circ}$ & 0.00 & 3.25 & 2.87 \\
\hline$(3,3)-\mathrm{Fe}$ & 2.59 & 1.94 & $144^{\circ}$ & 0.48 & 0.00 & 1.75 \\
\hline$(4,4)-\mathrm{Fe}$ & 2.77 & 2.22 & $126^{\circ}$ & 0.00 & 0.00 & 1.95 \\
\hline $\mathrm{C}_{120}-\mathrm{Fe}_{10}$ & 2.49 & 2.04 & $139^{\circ}$ & $<0.1$ & 2.28 & 1.82 \\
\hline \hline
\end{tabular}

TABLE I: Summary of properties for Fe structures. The bandgap is HOMO-LUMO for the toroidal molecule.

\begin{tabular}{|l||c|c|c|c|c|c||}
\hline \hline Structure & $\begin{array}{c}\mathrm{M}-\mathrm{M} \\
\AA\end{array}$ & $\begin{array}{c}\mathrm{M}-\mathrm{C} \\
\AA_{\text {min }}\end{array}$ & $\begin{array}{c}\mathrm{M}-\mathrm{M} \\
\text { Angle }\end{array}$ & $\begin{array}{c}\text { Gap } \\
\mathrm{eV}\end{array}$ & $\begin{array}{c}\text { Mom. } \\
\mu_{B} / \mathrm{M}\end{array}$ & $\begin{array}{c}\mathrm{E}_{\mathrm{B}} \\
\mathrm{eV} / \mathrm{M}\end{array}$ \\
\hline $\mathrm{Au}$ fcc & 2.88 & - & - & 0.00 & 0.00 & 3.05 \\
\hline $\mathrm{Au}$ chain & 2.83 & - & $57^{\circ}$ & 0.00 & 0.00 & 2.07 \\
\hline$(3,3)-\mathrm{Au}$ & 2.46 & 2.34 & $180^{\circ}$ & 0.00 & 0.00 & -6.80 \\
\hline$(4,4)-\mathrm{Au}$ & 2.52 & 2.56 & $156^{\circ}$ & 0.00 & 0.00 & -0.12 \\
\hline $\mathrm{C}_{120}-\mathrm{Au}_{10}$ & 2.43 & 2.00 & $143^{\circ}$ & $<0.1$ & 0.00 & -5.54 \\
\hline \hline
\end{tabular}

TABLE II: Summary of properties for Au structures. The bandgap is HOMO-LUMO for the toroidal molecule.

\begin{tabular}{||l||c|c|c|c|c|c||}
\hline \hline Structure & $\begin{array}{c}\mathrm{M}-\mathrm{M} \\
\AA\end{array}$ & $\begin{array}{c}\mathrm{M}-\mathrm{C} \\
\AA_{\text {min }}\end{array}$ & $\begin{array}{c}\mathrm{M}-\mathrm{M} \\
\text { Angle }\end{array}$ & $\begin{array}{c}\text { Gap } \\
\mathrm{eV}\end{array}$ & $\begin{array}{c}\text { Mom. } \\
\mu_{B} / \mathrm{M}\end{array}$ & $\begin{array}{c}\mathrm{E}_{\mathrm{B}} \\
\mathrm{eV} / \mathrm{M}\end{array}$ \\
\hline $\mathrm{Cu}$ fcc & 2.56 & - & - & 0.00 & 0.00 & 3.62 \\
\hline $\mathrm{Cu}$ chain & 2.42 & - & $59^{\circ}$ & 0.00 & 0.00 & 2.17 \\
\hline$(3,3)-\mathrm{Cu}$ & 2.46 & 2.24 & $177^{\circ}$ & 0.00 & 0.00 & -0.83 \\
\hline$(4,4)-\mathrm{Cu}$ & 2.47 & 2.69 & $168^{\circ}$ & 0.00 & 0.00 & 1.09 \\
\hline $\mathrm{C}_{120}-\mathrm{Cu}_{10}$ & 2.39 & 1.92 & $144^{\circ}$ & $<0.1$ & 0.00 & 0.01 \\
\hline \hline
\end{tabular}

TABLE III: Summary of properties for $\mathrm{Cu}$ structures. The bandgap is HOMO-LUMO for the toroidal molecule.

bcc iron, and it will be useful to determine to what degree, if any, this persists at finite temperatures. It is not clear what sort of chain structure and composite properties would result from the encapsulation of chains with symmetry at odds with that of the host torus.

\section{SUMMARY}

The azimuthal symmetry of encapsulated atomic metallic necklaces within toroidal carbon sheaths offers the prospect of synthesizing composites with novel electromagnetic properties that are not associated with straight, metal-encapsulated CNTs. As a first step in this direction, a particularly simple encapsulation was analyzed using density functional theory to determine its ground state geometry, structural stability, binding energy, electrical conductivity and magnetic moment. Of the four known classes of TCNs, a Type $1 \mathrm{C}_{120}$ molecule was chosen because it results in a computationally tractable composite, and only ten metal atoms were considered in order to take full computational advantage of the $\mathrm{D}_{5 d}$ symmetry of the torus. The TCN exhibits a positive, neutral and negative affinities for $\mathrm{Fe}, \mathrm{Cu}$ and $\mathrm{Au}$ atoms, respectively, and metal atom encapsulation resulted in linearly stable structures in all three cases. In contrast to Fe chains within $(3,3)$ and $(4,4)$ CNT's, Fe atoms within the $\mathrm{C}_{120}-\mathrm{Fe}_{10}$ molecule exhibit a net magnetic moment comparable to bcc Fe.

All toroidal nanotubes should all be capable of serving as sheathing hosts for atomic metal necklaces with novel electromagnetic properties. The possibility of en- 
capsulating metal chains within the $\mathrm{C}_{60}$-coalesced tori of Types 3 and 4 is of particular interest because these are structures that have already been synthesized [6], [7]. For Type $3 \mathrm{TCNs}$, a reasonable strategy might be to encapsulate $\mathrm{Fe}$ atoms within the $\mathrm{C}_{60}$ molecules prior to coalescing them into a toroid. Sufficiently long CNTs with embedded metal atoms could also be deformed to synthesize Type 2 TCN composites. Both approaches would admit the construction of alloyed necklaces as well-the one-dimensional, atomic analog of powder metallurgy.

* Electronic address: mlusk@mines.edu

[1] Dunlap, B. I. Phys. Rev. B 46(3), 1933-1936 Jul (1992).

[2] Ihara, S., Itoh, S., and Kitakami, J.-i. Phys. Rev. B 47(19), 12908-12911 May (1993).

[3] Itoh, S., Ihara, S., and Kitakami, J.-i. Phys. Rev. B 47(3), 1703-1704 Jan (1993).

[4] Kurby, E., Mallion, R. B., and Pollak, P. J. Chem. Soc. Farad. T. 89, 1945-1953 (1993).

[5] Meunier, V., Lambin, P., and Luca, A. Phys. Rev. B 57(23), 14886-14890 Jun (1998).

[6] Rodríguez-Manzo, J. A., López-Urías, F., Terrones, M., and Terrones, H. Nano Lett. 4(11), 2179-2183 (2004).

[7] Terrones, H., Lopez-Urias, F., Munoz-Sandoval, E., Rodriguez-Manzo, J., Zamudio, A., Elias, A., and Terrones, M. Solid State Sci. 8(3-4), 303-320 (2006).

[8] Terrones, H., Terrones, M., Hernández, E., Grobert, N., Charlier, J.-C., and Ajayan, P. M. Phys. Rev. Lett. 84(8), 1716-1719 Feb (2000).

[9] Chen, N., Lusk, M., van Duin, A., and Goddard, W. Phys. Rev. B 72, 085416 (2005).

[10] London, F. J Phys.-Paris 8, 397-409 (1937).

[11] Lu, J. P. Phys. Rev. Lett. 74(7), 1123-1126 Feb (1995).

[12] Haddon, R., Schneemeyer, L., Waszczak, J., Glarum, S., Tycko, R., Dabbagh, G., Kortan, A., Muller, A., Mujsce, A., Rosseinsky, M., Zahurak, S., Makhija, A., Thiel, F., Raghavachari, K., Cockayne, E., and Elser, V. Nature 350, 46-47 (1991).
[13] Haddon, R. Nature 388, 31-32 (1997).

[14] Lin, M. F. and Chuu, D. S. Phys. Rev. B 57(11), 67316737 Mar (1998).

[15] Liu, L., Guo, G. Y., Jayanthi, C. S., and Wu, S. Y. Phys. Rev. Lett. 88(21), 217206 May (2002).

[16] Yanson, A. I., Bollinger, G. R., van den Brom, H. E., Agra, N., and van Ruitenbeek, J. M. Nature 395, 783785 (1998).

[17] Ohnishi, H., Kondo, Y., and Takayanagi, K. Nature 395, 780-783 (1998).

[18] Sanchez-Portal, D., Artacho, E., Junquera, J., Ordejo, P., Garcia, A., and Soler, J. Phys. Rev. Lett. 83(19), 3884-3887 Nov (1999).

[19] Rollmann, G., Entel, P., and Sahoo, S. Comp. Mater. Sci. 35(3), 275-278 (2006).

[20] Jain, P. Struc. Chem. 16(4), 421-426 Aug (2005).

[21] Rahman, M. M., Kisaku, M., Kishi, T., Matsunaka, D., Diño, W., Nakanishi, H., and Kasal, H. J. Phys-Condens. Mat. 16, S5755-S5758 (2004).

[22] Weissmann, M., García, G., Kiwi, M., Ramírez, R., and Fu, C. Phys. Rev. B 73, 125435 (2006).

[23] Delley, B. J. Chem. Phys. 113(18), 7756-7764 November (2000).

[24] Perdew, J. P. and Wang, Y. Phys. Rev. B 45(23), 1324413249 Jun (1992).

[25] Reddy, B. V. and Khanna, S. N. Phys. Rev. Lett. 93(6), 068301 (2004).

[26] Ackland, G., D.J.Bacon, Calder, A., and Harry, T. Phil. Mag. A 75, 713 (1997).

[27] Kittel, C. Introduction to Solid State Physics, 7th Edition. Wiley, New Jersey, (1996).

[28] Acet, M., Zähres, H., Wassermann, E. F., and Pepperhoff, W. Phys. Rev. B 49(9), 6012-6017 Mar (1994).

[29] Garcia-Suarez, V., Newman, C., Lambert, C., Pruneda1, J., and Ferrer, J. Eur. J. Phys. B 40, 371-377 (2006).

[30] Yanson, A. Ph.D. Thesis, Leiden Institute of Physics (1975).

[31] Kisaku, M., Rahman, M., Kishi, T., Matsunaka, D., Roman, T., Dino, W., Nakanishi, H., and Kasai, H. Jpn. J. Appl. Phys. 44(2), 882-888 (2005).

[32] Skumanich, A. Chem. Phys. Lett. 182(5), 486-490 (1991). 